\title{
DAX-1 Inhibits Hepatocellular Carcinoma Proliferation by Inhibiting $\beta$-Catenin Transcriptional Activity
}

\author{
Hong-Lei Jiang Dong Xu Hao Yu Xu Ma Guo-Fu Lin Dong-Yan Ma \\ Jun-Zhe Jin
}

The Fourth Affiliated Hospital of China Medical University, Shenyang, China

\author{
Key Words \\ DAX1 • $\beta$-Catenin $\cdot$ Cell proliferation $\cdot$ Hepatocellular carcinoma
}

\begin{abstract}
Background/Aims: Hepatocellular carcinoma (HCC) represents the most common type of liver cancer. DAX1 (dosage-sensitive sex reversal adrenal hypoplasia congenital critical region on $X$ chromosome, gene 1 ), an atypical member of the nuclear receptor family due to lack of classical DNA-binding domains, has been known for its fundamental roles in the development, especially in the sex determination and steroidogenesis. Previous studies also showed that DAX-1 played a critical role in endocrine and sex steroid-dependent neoplasms such as adrenocortical, pituitary, endometrial, and ovarian tumors. However, its biological roles in the development of HCC remain largely unexplored. Methods: Real-time PCR and Western blot were used to detect the expression of DAX-1 in HCC tissues and cell lines. Immunoprecipitation (IP) assay was used to show the interaction between DAX-1 and $\beta$-Catenin. Small interfering RNA (siRNA) was used to silence the expression of DAX-1. BrdU incorporation and Cell-cycle assays were used to detect the role of DAX-1 in HCC cells proliferation. Migration and invasion assays were carried out to test the metastasis ability of DAX-1 in HCC cells. Results: In the present study, we found that mRNA and protein levels of DAX-1 were down-regulated in HCC tissues and cell lines. Furthermore, overexpression of DAX-1 could inhibit while its knockdown using small interfering RNA promoted cell proliferation in several HCC cell lines. At the molecular level, we demonstrated that DAX-1 could interact with $\beta$-Catenin and attenuate its transcriptional activity. Conclusion: Therefore, our results suggest a previously unknown DAX$1 / \beta$-Catenin molecular network controlling HCC development.

Copyright $\odot 2014$ S. Karger AG, Basel

Jun-Zhe Jin M.D.

The Fourth Affilated Hospital of China Medical University, Department of Hepatobiliary Surgery, The No.4, Chongshan east Road, Huang gu District, Shenyang, Liaoning, 110032, (China)

Tel. +86 024-62043517, Fax +86 24-62255001, E-Mail jin_junzhe@126.com
\end{abstract}




\section{Introduction}

Hepatocellular carcinoma (HCC) has become one of the most common malignancy and lethal neoplasm in china [1]. Although roles of multiple oncogenes and (or) tumor suppressor in the development of HCC have been characterized [2], its molecular mechanisms remain to be elucidated.

DAX-1 (dosage-sensitive sex reversal, adrenal hypoplasia critical region, on chromosome $X$, gene 1), is an orphan nuclear receptor and plays fundamental roles in the steroidogenesis $[3,4]$. In human, several reports indicate that DAX-1 gene mutations are tightly linked to congenital adrenal hypoplasia and primary adrenal insufficiency $[5,6]$. Besides, DAX1 was also identified to participate in human cancers, including adrenocortical, pituitary, endometrial, and ovarian tumors [7]. For instance, DAX-1 overexpression could inhibit estrogen-dependent breast cancer cell proliferation through down-regulating aromatase expression [8]. Besides, DAX-1 could interact with and inhibit the nuclear localization of androgen receptor in prostate cancer [9]. Therefore, abnormal expression of DAX-1 might be involved in the pathogenesis of several human cancers. However, whether DAX-1 participates in the progression of HCC remains largely unknown.

In the present study, we found that DAX-1 expression was down-regulated in HCC tissues and cell lines. Overexpression of DAX-1 inhibited, while its deficiency using small interfering RNA (siRNA) promoted proliferation, invasion and metastasis of hepatoma cells. Furthermore, we discovered that DAX-1 played a role in HCC development through regulation of Wnt/ $\beta$-Catenin signaling. Therefore, our results suggest a novel role of DAX-1 in the regulation of HCC development.

\section{Material and Methods}

\section{Tissue samples}

Primary hepatocellular carcinoma tissues, adjacent normal liver tissues were collected from routine therapeutic surgery at our department. All samples were obtained with informed consent and approved by our hospital.

\section{Cell culture}

All cell lines used were purchased from The Cell Bank of Type Culture Collection of Chinese Academy of Sciences (CAS, Shanghai, China), and cultured in Dulbecco modified Eagle's medium supplemented with $10 \%$ fetal calf serum, $100 \mathrm{IU} / \mathrm{ml}$ penicillin and $100 \mathrm{mg} / \mathrm{ml}$ streptomycin. Cells were incubated at $37^{\circ} \mathrm{C}$ under $5 \% \mathrm{CO}_{2}$ humidified atmosphere.

\section{BrdU incorporation and Cell-cycle assays}

For BrdU incorporation assays, a cell proliferationenzyme-linked immunosorbent assay kit (Beyotime, Shanghai, China) was employed to analyze the incorporation of BrdU during DNA synthesis following the manufacturer's protocols. For cell-cycle assays, cells were labeled for $15 \mathrm{~min}$ with PI and immediately analyzed by flow cytometry. Histograms represent the percentage of cells in each phase of the cell cycle (G0/G1, S and G2/M).

\section{Cell migration and invasion assays}

Cell migration and invasion were analyzed using Transwell migration and extracellular matrix-coated invasion chambers (Millipore, CA, USA) according to the manufacturer's instructions. In short, cells were plated into the top well of a Transwell migration chamber (for migration assay) or an extracellular matrixcoated invasion chamber (for invasion assay). After 24 hours incubation, the non-migrating/non-invading cells were removed with a cotton swab, and the migrating/invading cells on the underside of the membrane were stained with cell stain solution (Millipore) for 15 minutes. After washed 2 times with PBS, the stain of each membrane was removed with $100 \mathrm{~mL}$ extraction buffer (Millipore) and quantitated with a colorimetric microplate reader at $570 \mathrm{~nm}$. 
Jiang et al.: DAX-1 Inhibits Hepatocellular Carcinoma Proliferation

SiRNA, RNA extraction and Real-time Analysis

Hep3B Cells were seeded on to 6-well plates then transfected with 20nM siGENOME non-targeting siRNA, human DAX-1 (5'-GCACGUGGCU CCUUAGCGAU-3', Dharmacon, USA). Total RNAs were isolated from tissues or cells by TRIzol reagent, and reverse transcriptions were performed by Takara RNA PCR kit (Takara, Dalian, China) following the manufacturer's instructions. In order to quantify the transcripts of the interest genes, real-time PCR was performed using a SYBR Green Premix Ex Taq (Takara, Japan) on Light Cycler 480 (Roche, Switzerland). PCR conditions included an initial holding period at $95^{\circ} \mathrm{C}$ for $5 \mathrm{~min}$, followed by a twostep PCR program consisting of $95^{\circ} \mathrm{C}$ for $5 \mathrm{~s}$ and $60^{\circ} \mathrm{C}$ for $30 \mathrm{~s}$ for 45 cycles. The primer sequences were listed as following: DAX-1 (Sense:5'-AGCACAAATCAAGCGCAGG-3',Antisense:5'-GAAGCGCAGCGTCTTCAAC-3'); c-Myc(Sense:5'-GGCTCCTGGCAAAAGGTCA-3', Antisense:5'- CTGCGTAGTTGTGCTGATGT-3');CyclinD1(Sense:5'-GCTGCGAAGTGGAAACCATC-3',Antisense:5'-CCTCCTTCTGCACACATTTGAA-3'); Cyclin E (Sense:5'- GCCAGCCTTGGGACAATAATG-3',Antisense:5'-CTTGCACGTTGAGTTTGGGT-3')

Transient Transfections and Luciferase assays

TOP-flash and FOP-flash luciferase reporters were gifts from Dr. Xiang Yu (Institute of Neuroscience, SIBS, CAS, Shanghai). All the transient transfections were performed by Lipofectamine 2000 (Invitrogen), according to the manufacturer's instructions. For luciferase reporter assays, Hep3B cells were seeded in 24-well plates and transfected with the indicated plasmids. Cells were harvested $48 \mathrm{hr}$ after transfection. Luciferase activities were measured using the Dual Luciferase Reporter Assay System (Promega, USA).

Immunoprecipitation and Western blot

Cells were harvested, resuspended in lysis buffer containing $50 \mathrm{mM}$ Tris- $\mathrm{HCl}$ (pH 7.4), $150 \mathrm{mM} \mathrm{NaCl}$, $1 \mathrm{mM}$ EDTA, 0.5\% TRITON X-100)and protease inhibitors. Lysates were incubated with $4 \mu \mathrm{g}$ of indicated antibodies or IgG overnight at $4^{\circ} \mathrm{C}$ with gentle inversion. Protein $\mathrm{A} / \mathrm{G}$ beads were added for additional 6 $\mathrm{hr}$ at $4^{\circ} \mathrm{C}$. Beads containing immune complexes were washed with $0.8 \mathrm{ml}$ ice cold lysis buffer for three times. Precipitates were denatured in Laemmli (gel loading) buffer at $99^{\circ} \mathrm{C}$ for $10 \mathrm{~min}$. For western blot analysis, cells were harvested by trypsinization, lysed in 2 x Laemmli buffer (100 mM Tris-HCl at pH 6.8, $200 \mathrm{mM} \mathrm{DTT}, 4 \% \mathrm{SDS}[\mathrm{w} / \mathrm{v}], 20 \%$ glycerol, $0.05 \%$ bromophenol blue), denatured for $10 \mathrm{~min}$ at $80^{\circ} \mathrm{C}$, sheared with an insulin syringe, and resolved on SDS/PAGE gels. After immunoblotting, the membranes were blocked in PBS/0.1\% Tween-20 with 5\% nonfat dry milk, and primary antibodies were incubated in PBS $/ 0.1 \%$ Tween-20 with $0.1 \%-5 \%$ nonfat dry milk. Antibodies directed against DAX-1, $\beta$-Catenin, Cyclin D1 and Cyclin E were purchased from Abcam Company (USA). Anti-GAPDH was obtained from Santa Cruz Biotechnology (USA).

\section{Xenograft tumor growth in nude mice}

4 weeks old, male BALB/c nude mice were purchased from the Experimental Animal Center of the China Medical University. Approximately $6 \times 10^{5}$ Hep3B cells stably overexpressing DAX-1 or empty vector were injected subcutaneously to the skin under the front legs of the mice. The mice were observed over 4 weeks for tumor formation. Then all the mice were euthanized and the tumor tissues were removed by surgical excision. The tumors were recovered and the wet weights of each tumor were determined. The experiments were performed using six mice per group.

\section{Statistical Analysis}

All data are presented as mean \pm SEM. Statistical differences were determined by a two-tailed $t$ test. Statistical significance is displayed as $*(\mathrm{P}<0.05),{ }^{* *}(\mathrm{P}<0.01)$ or ${ }^{* * *}(\mathrm{P}<0.001)$.

\section{Results}

Down-regulation of DAX-1 in HCC tissues or cells

First, DAX-1 expression was analyzed in 25 paired of HCC and adjacent non-tumor liver tissues using real-time PCR and western blot. As a result, our results showed that DAX-1 was significantly down-regulated in cancer tissues (Fig. 1A-1B). To further determine the relationship between DAX-1 and HCC, several liver cell lines were analyzed. As show in in the 


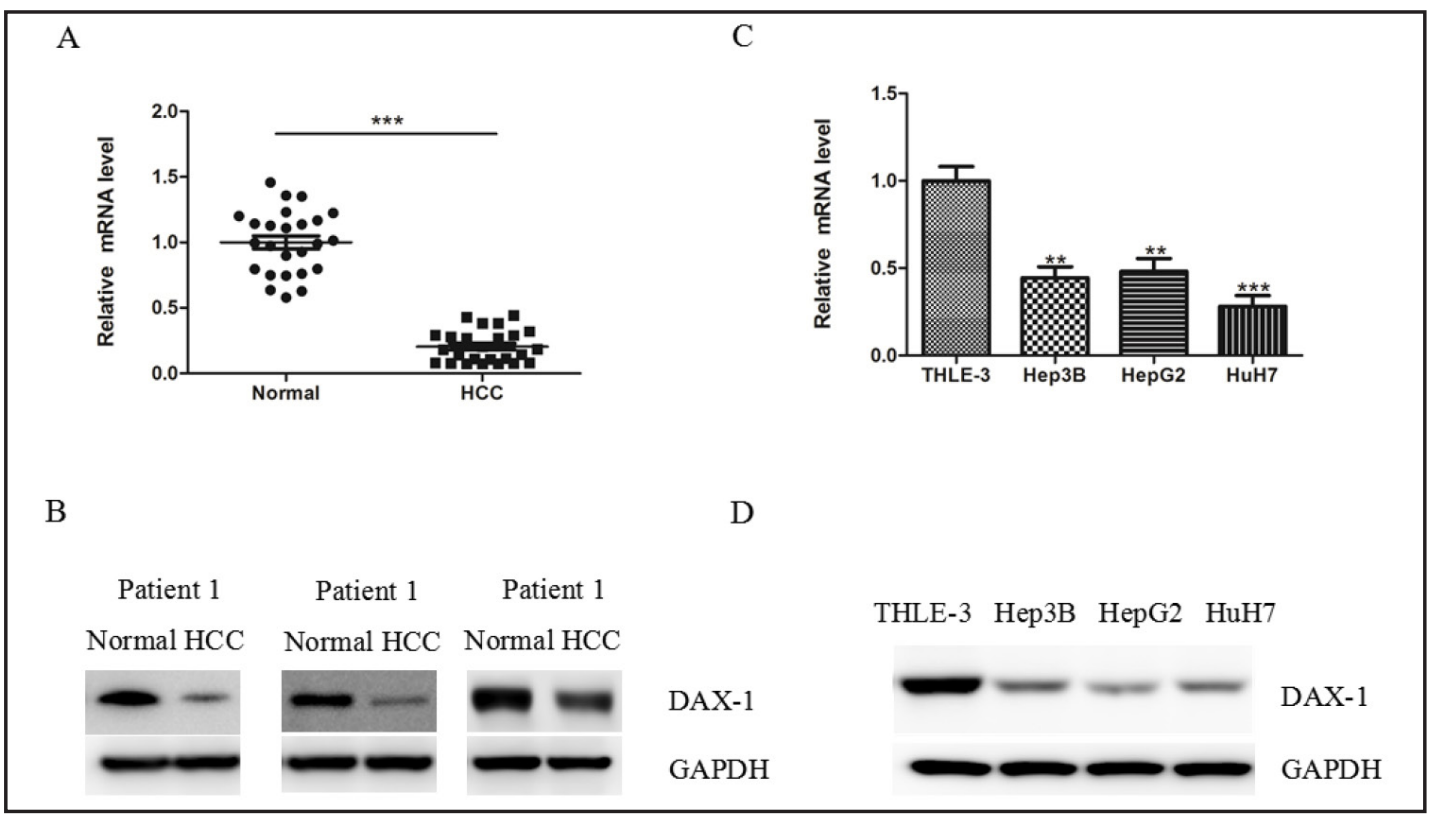

Fig. 1. Down-regulation of DAX-1 in HCC tissues and cell lines. (A-B) mRNA and representative protein levels of DAX-1 were analyzed by real-time PCR (A) and western blot (B) in HCC tissues and normal tissues. (C-D) The mRNA and protein levels of DAX-1 were determined in normal liver cells (THLE-3 cells) and several liver cancer cell lines, including HepG2, Hep3B, and HuH-7 cells.

Fig. 1C and 1D, we also observed the lower expression of DAX-1 in three liver cancer cell lines (HepG2, Hep3B, and HuH-7), compared with normal liver cell line (THLE-3), indicating that DAX-1 expression was down-regulated in HCC tissues and cell lines.

\section{Effect of DAX-1 on HCC cell proliferation}

To elucidate the functional role of DAX-1 in the HCC development, adenoviruses containing DAX-1 or empty vector (EV) were transfected into Hep3B cells (Fig. 2A). As shown in Figure 3B, DAX-1 overexpression led to a significant decrease in cell number of all cells tested (Fig. 2B). Consistently, cell proliferation ability was also impaired by way of bromodeoxyuridine (BrdU) analysis (Fig. 2C). Moreover, DAX-1 overexpression resulted in a significant increase in G0/G1 phase and decrease in S phase (Fig. 2D). Similar results were also observed in HepG2 cells (Fig. 2E-2G).

Next, small interfering RNA (siRNA) targeting DAX-1 gene or negative control was employed to knockdown endogenous DAX-1 expression in Hep3B cells (Fig. 3A). As a result, down-regulation of DAX-1 led to a marked increase in cell number and proliferative ability in these cells (Fig. 3B-3C). DAX-1 deficiency also resulted in a decrease in G0/G1 phase and increase in S phase (Fig. 3D). Taken together, our results suggest that DAX-1 might be an important negative regulation of cell proliferation.

\section{Effect of DAX-1 on HCC cell metastasis and invasion}

Next, cell migration and invasion assays were carried out in Hep3B cells, which demonstrated that DAX-1 overexpression significantly inhibited, while its deficiency promoted the in vitro cell migration and invasion abilities (Fig. 4A-4D).

DAX-1 overexpression inhibits tumor growth in vivo

To further investigate the function of DAX-1 on HCC growth in vivo, Hep3B cells with stable overexpression of DAX-1 were generated and injected subcutaneously into nude mice. The tumor growth was closely monitored for another 5 weeks. As compared with control, the 
Fig. 2. DAX-1 overexpression inhibits HCC cell proliferation. (A) DAX-1 expression was determined by western blot in Hep3B cells transfected with adenoviruses containing empty vector (EV) or DAX-1. (B) The growth curve of Hep3B cells transfected with empty vector (EV) or DAX-1. (C) The cell proliferative potential (BrdU) was determined in Hep3B cells transfected with empty vector (EV) or DAX-1. (D) The cell cycle phase of Hep3B cells transfected with empty vector (EV) or DAX-1 were analyzed

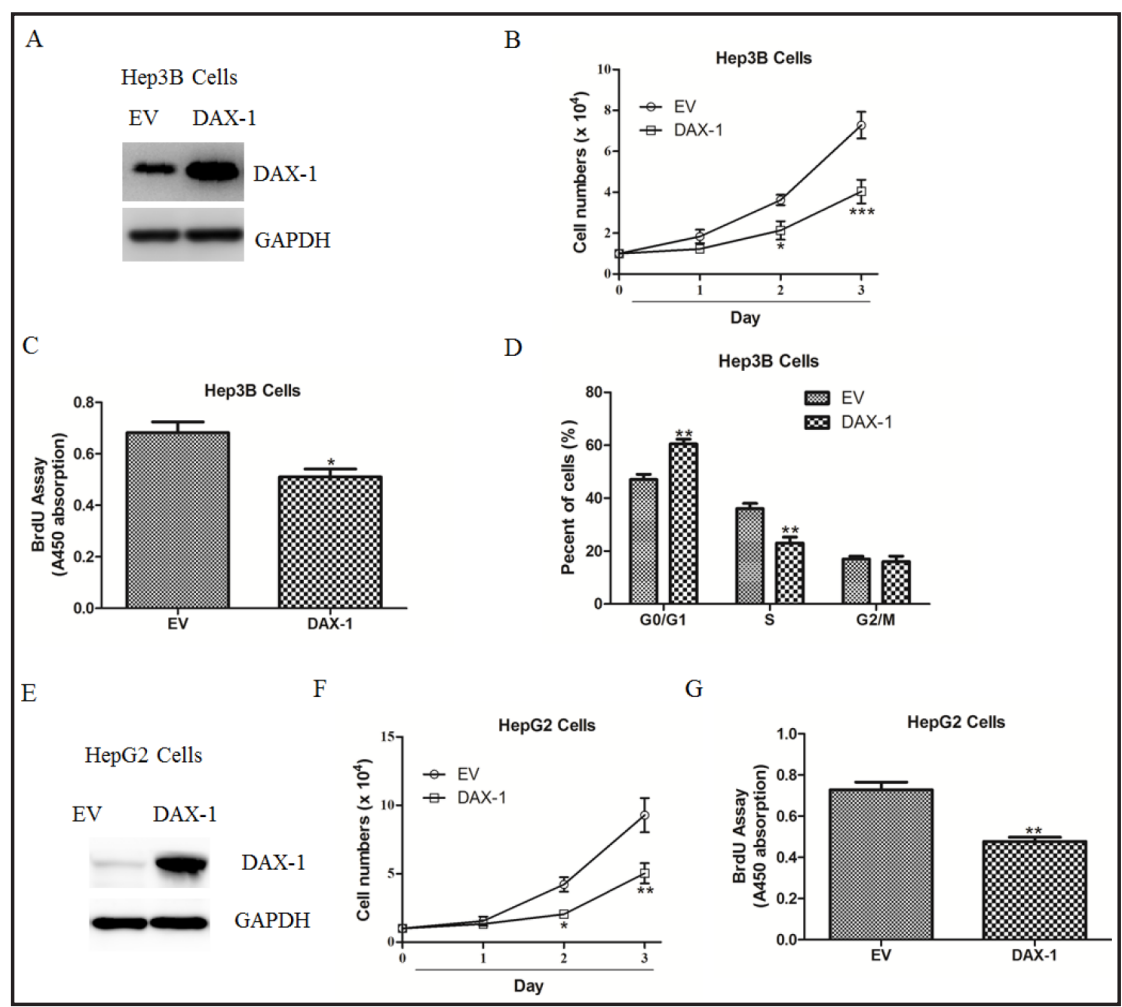

by flow cytometry. Histograms represent the percentage of cells in each phase of the cell cycle (G0/G1, S and G2/M). (E) DAX-1 expression was determined by western blot in HepG2 cells transfected with adenoviruses containing empty vector (EV) or DAX-1. (F) The growth curve of HepG2 cells transfected with empty vector (EV) or DAX-1. (G) The cell proliferative potential (BrdU) was determined in HepG2 cells transfected with empty vector $(\mathrm{EV})$ or DAX-1.

Fig. 3. DAX-1 deficiency promotes HCC cell proliferation. (A) DAX-1 expression was determined by western blot in Hep3B cells transfected with siRNA oligos targeting DAX-1 or scramble negative controls (NC). (B) The growth curve of Hep3B cells transfected with transfected with siRNA oligos targeting DAX-1 or negative controls (NC). (C) The cell proliferative potential (BrdU) was determined

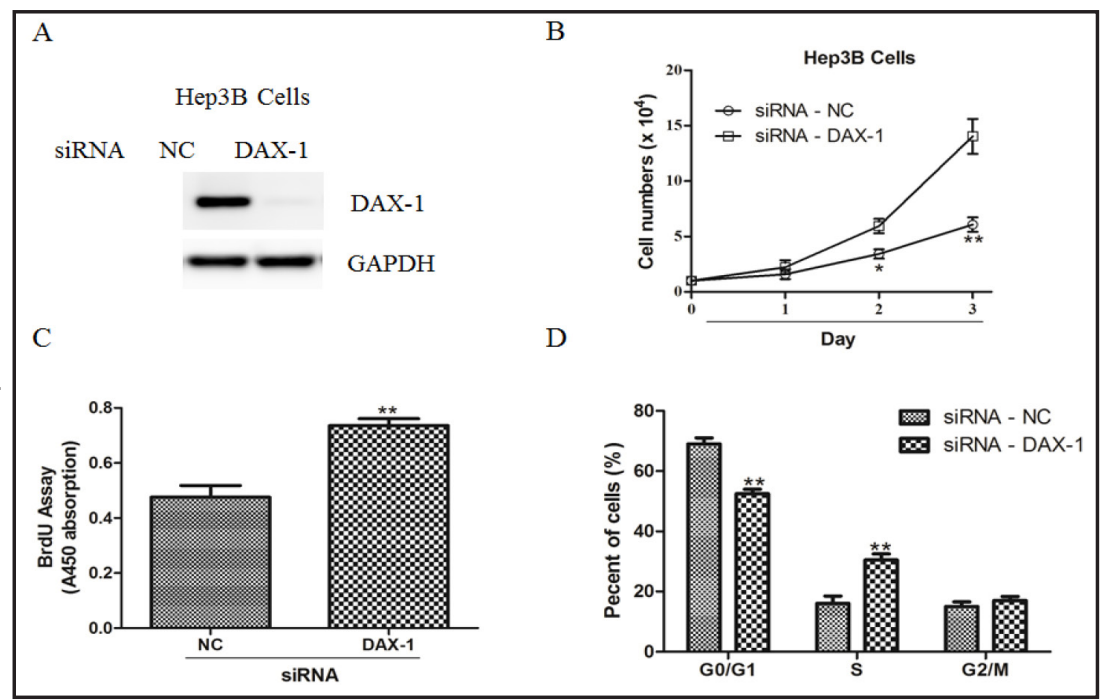

in Hep3B cells transfected with DAX-1 siRNA or negative controls (NC). (D) The cell-cycle analysis was determined in Hep3B cells transfected with DAX-1 siRNA or negative controls (NC).

tumor size and volume were markedly decreased in DAX-1-overexpressed tumors compared to control tumors (Fig. 5A-5B). The average tumor weight was also significantly increased (Fig. 5C), suggesting that DAX-1 could suppress tumor growth in vivo. 
Fig. 4. Roles of DAX-1 in the HCC cell metastasis and invasion. (A-B) The migration and invasion abilities of Hep3B cells. After transfected with empty vector (EV) or DAX-1 for 24 hours, cells were seeded in Transwell filters (A) or ECM gel-coated Transwell culture chambers (B) and incubated for another 24

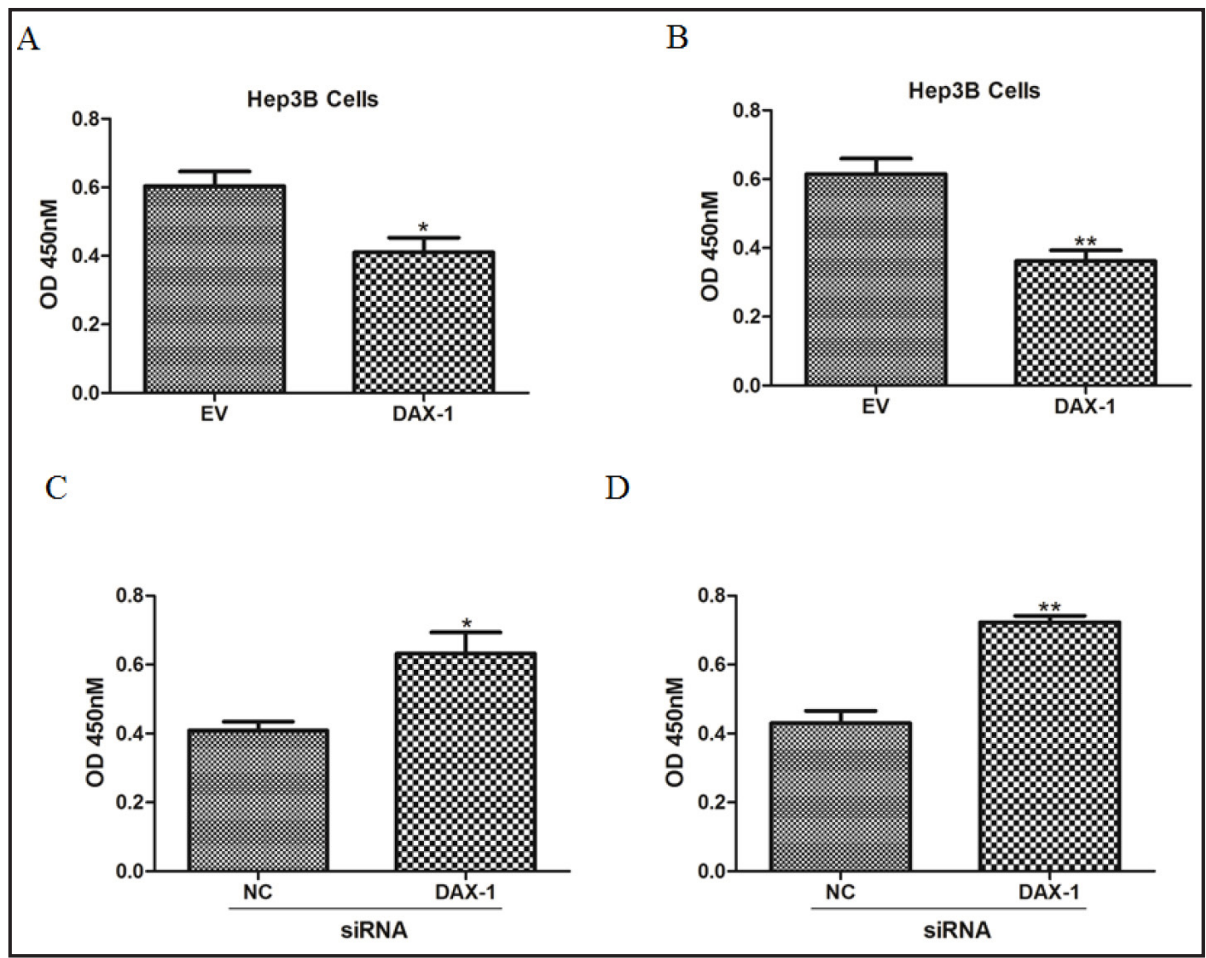

hours, then the Transwell migration assay (A) or invasion assay (B) were conducted. (C-D) The cell invasion (C) and migration (D) abilities in Hep3B cells transfected with DAX-1 siRNA or negative controls (NC).

A

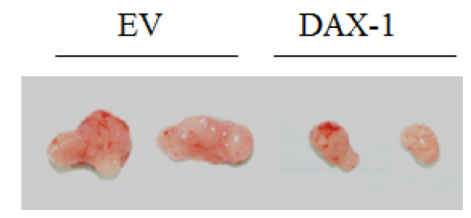

Fig. 5. DAX-1 overexpression inhibits tumor growth in the nude mice. (A-C) Hep3B cells EV-transfected or stably transfected with DAX-1 were injected into nude mice and followed up for tumorigenesis. Representative pictures (A), tumor volume (B) and weights (C) were taken 4 weeks after injection.
B

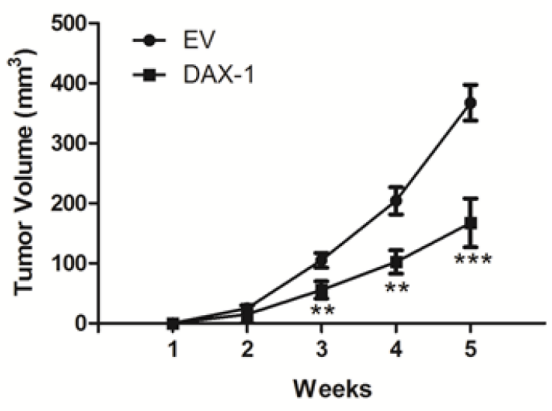

C

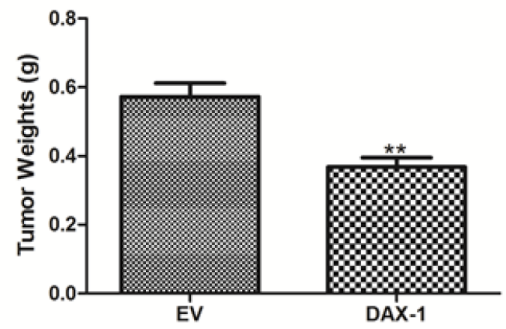

Negative regulation of Wnt/ $\beta$-Catenin signaling by DAX-1 in HCC cells

Previous studies have revealed that the Wnt/ $\beta$-Catenin signaling plays an essential role in HCC development $[10,11]$. We asked whether overexpression or knockdown of DAX-1 


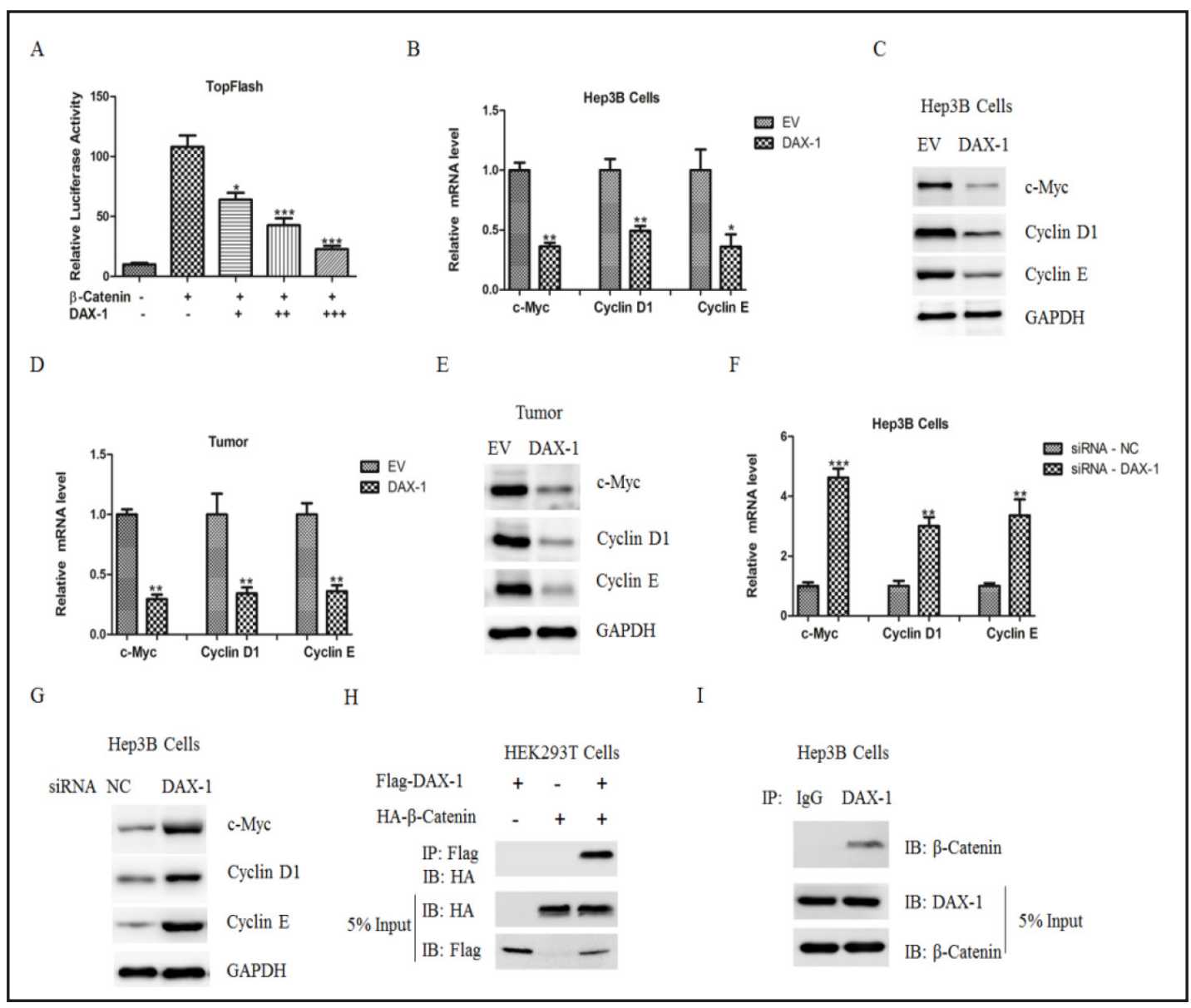

Fig. 6. DAX-1 regulation of Wnt/ $\beta$-Catenin signaling in Hep3B cells. Activity of the TopFlash reporter gene in Hep3B cells transfected with $\beta$-Catenin in the absence or presence of DAX-1 $(100,200,400 \mathrm{ng})$ or empty vector. (B-C) Expression of C-myc, Cyclin D1 and Cyclin E were analyzed by real-time PCR and western blot in Hep3B cells. (D-E) Expression levels of C-myc, Cyclin D1 and Cyclin E were analyzed by real-time PCR (D) and western blot (E) in the tumors from nude mice. (F-G) Expression levels of C-myc, Cyclin D1 and Cyclin E were analyzed by real-time PCR (F) and western blot $(G)$ in Hep3B cells transfected with DAX-1 siRNA or negative controls (NC). (H) HEK293T cells were infected with Flag-DAX-1, HA- $\beta$-Catenin or empty vectors. HA- $\beta$-Catenin was immunoprecipitated (IP) from total cell lysates with anti-Flag antibodies. The amount of immunoprecipitated HA- $\beta$-Catenin was determined by western blot analysis. (I) The endogenous interaction of DAX-1 and $\beta$-Catenin was determined in Hep3B cells.

may affect Wnt/ $\beta$-Catenin signaling. We used TOP-flash and FOP-flash reporter system to measure $\beta$-catenin-mediated transcriptional activity. As shown in Figure 6A, overexpression of DAX-1 reduced the luciferase activity. Besides, expression levels of its downstream target genes [12], such as C-myc, Cyclin D1 and Cyclin E, were also down-regulated (Fig. 6B-6C). In agreement, their expression was also decreased in tumors overexpressing DAX-1 (Fig. 6D6E). In contrast, DAX-1 depletion dramatically led to an accumulation of C-myc, Cyclin D1 and Cyclin E (Fig. 6F-6G).

In order to investigate how DAX-1 regulates $\beta$-catenin transcriptional activity, we examined the interaction of these two proteins through in vivo binding assays. Indeed, our co-immunoprecipitation (Co-IP) assays showed that DAX-1 could interact with $\beta$-Catenin in HEK293T and Hep3B cells (Fig. 6H-6I), suggesting that DAX-1 may inhibit the transcriptional activity of $\beta$-Catenin through their protein-protein interaction. 


\section{Discussion}

Previous studies have shown that DAX-1 could act as a corepressor for several transcription factors and nuclear receptors. For instance, DAX-1 could inhibit hepatic gluconeogenic enzyme gene expression through negatively regulating the activity of hepatocyte nuclear factor 4 (HNF4) [13]. Besides, DAX-1 was shown to interact with liver $\mathrm{X}$ receptor (LXR) and farnesoid X receptor (FXR) to regulate hepatic triglyceride and bile acid metabolism, respectively $[14,15]$. A recent study also suggest that DAX-1 participated in the acetaminophen (APAP)-induced hepatotoxicity through regulation of pregnane $\mathrm{X}$ receptor (PXR) and constitutive androstane receptor (CAR) [16]. Therefore, the roles and molecular mechanisms of DAX-1 in livers might be diverse and complicated, which remains to be explored in the future.

In the present study, our findings uncovered an important role for DAX-1 in controlling HCC development via regulating $\beta$-Catenin activity, suggesting that DAX-1 might be a tumor suppressor in the HCC development. The nuclear localization and activation of $\beta$-catenin represents the common hallmark in various human caner tissues [17]. Usually, $\beta$-catenin activity is tightly regulated by a multi-protein complex, including the tumor suppressor APC, the scaffolding protein Axin, and the phosphokinases GSK-3 $\beta$ [18]. Upon activation, the complex is destabilized, allowing for cytoplasmic $\beta$-catenin retention with subsequent nuclear translocation [19]. Previous reports have demonstrated that the repressive roles of DAX-1 required the corepressor NCOR and histone deacetylase (HDAC) [20, 21]. Therefore, we speculate that DAX-1 might inhibit $\beta$-catenin transcriptional activity through recruitment of these corepressors. However, the precise roles and mechanisms of DAX-1 in HCC need to be further investigated using other models, such as liver-specific DAX-1 knockout mice.

In summary, our data provide the evidence that DAX-1 could inhibit HCC cell growth in vitro and in vivo, implicating an important role of DAX-1 and suggesting that it might be a potential therapeutic strategy for HCC.

\section{Disclosure Statement}

None

\section{Acknowledgement}

This work was supported by the Science and Technology Program of LiaoNing (2012225019).

\section{References}

1 Song P, Gao J, Inagaki Y, Kokudo N, Hasegawa K, Sugawara Y, Tang W: Biomarkers: Evaluation of screening for and early diagnosis of hepatocellular carcinoma in japan and china. Liver cancer 2013;2:31-39.

2 Li Y, Tang ZY, Hou JX: Hepatocellular carcinoma: Insight from animal models. Nat Rev Gastroenterol Hepatol 2012;9:32-43.

3 McCabe ER: Dax1: Increasing complexity in the roles of this novel nuclear receptor. Mol Cell Endocrinol 2007;265-266:179-182.

4 El-Khairi R, Martinez-Aguayo A, Ferraz-de-Souza B, Lin L, Achermann JC: Role of dax-1 (nr0b1) and steroidogenic factor-1 (nr5a1) in human adrenal function. Endocr Dev 2011;20:38-46.

$>5$ Jadhav U, Harris RM, Jameson JL: Hypogonadotropic hypogonadism in subjects with dax1 mutations. Mol Cell Endocrinol 2011;346:65-73. 
6 Li N, Liu R, Zhang H, Yang J, Sun S, Zhang M, Liu Y, Lu Y, Wang W, Mu Y, Ning G, Li X: Seven novel dax1 mutations with loss of function identified in chinese patients with congenital adrenal hypoplasia. J Clin Endocrinol Metab 2010;95:E104-111.

7 Lalli E, Alonso J: Targeting dax-1 in embryonic stem cells and cancer. Expert Opin Ther Targets 2010;14:169-177.

8 Lanzino M, Maris P, Sirianni R, Barone I, Casaburi I, Chimento A, Giordano C, Morelli C, Sisci D, Rizza P, Bonofiglio D, Catalano S, Ando S: Dax-1, as an androgen-target gene, inhibits aromatase expression: A novel mechanism blocking estrogen-dependent breast cancer cell proliferation. Cell Death Dis 2013;4:e724.

-9 Nakamura Y, Suzuki T, Arai Y, Sasano H: Nuclear receptor dax1 in human prostate cancer: A novel independent biological modulator. Endocr J 2009;56:39-44.

10 Moeini A, Cornella H, Villanueva A: Emerging signaling pathways in hepatocellular carcinoma. Liver cancer 2012;1:83-93.

11 Shiraha H, Yamamoto K, Namba M: Human hepatocyte carcinogenesis (review). Int J Oncol 2013;42:11331138.

12 Dahmani R, Just PA, Perret C: The wnt/beta-catenin pathway as a therapeutic target in human hepatocellular carcinoma. Clin Res Hepatol Gastroenterol 2011;35:709-713.

13 Nedumaran B, Hong S, Xie YB, Kim YH, Seo WY, Lee MW, Lee CH, Koo SH, Choi HS: Dax-1 acts as a novel corepressor of orphan nuclear receptor hnf4alpha and negatively regulates gluconeogenic enzyme gene expression. J Biol Chem 2009;284:27511-27523.

14 Nedumaran B, Kim GS, Hong S, Yoon YS, Kim YH, Lee CH, Lee YC, Koo SH, Choi HS: Orphan nuclear receptor dax-1 acts as a novel corepressor of liver $\mathrm{x}$ receptor alpha and inhibits hepatic lipogenesis. J Biol Chem 2010;285:9221-9232.

15 Li J, Lu Y, Liu R, Xiong X, Zhang Z, Zhang X, Ning G, Li X: Dax1 suppresses fxr transactivity as a novel corepressor. Biochem Biophys Res Commun 2011;412:660-666.

16 Li M, Yang Y, He ZX, Zhou ZW, Yang T, Guo P, Zhang X, Zhou SF: Microrna-561 promotes acetaminopheninduced hepatotoxicity in hepg2 cells and primary human hepatocytes through downregulation of the nuclear receptor corepressor dosage-sensitive sex-reversal adrenal hypoplasia congenital critical region on the x chromosome, gene 1 (dax-1). Drug Metab Dispos 2014;42:44-61.

17 Anastas JN, Moon RT: Wnt signalling pathways as therapeutic targets in cancer. Nat Rev Cancer 2013;13:11-26.

18 Niehrs C: The complex world of wnt receptor signalling. Nat Rev Mol Cell Biol 2012;13:767-779.

19 Zeki SS, Graham TA, Wright NA: Stem cells and their implications for colorectal cancer. Nat Rev Gastroenterol Hepatol 2011;8:90-100.

-20 Crawford PA, Dorn C, Sadovsky Y, Milbrandt J: Nuclear receptor dax-1 recruits nuclear receptor corepressor n-cor to steroidogenic factor 1. Mol Cell Biol 1998;18:2949-2956.

21 Ehrlund A, Anthonisen EH, Gustafsson N, Venteclef N, Robertson Remen K, Damdimopoulos AE, Galeeva A, Pelto-Huikko M, Lalli E, Steffensen KR, Gustafsson JA, Treuter E: E3 ubiquitin ligase rnf31 cooperates with dax-1 in transcriptional repression of steroidogenesis. Mol Cell Biol 2009;29:2230-2242. 